\title{
Integration of transcriptomic data identifies key hallmark genes in hypertrophic cardiomyopathy
}

Jing $X u^{1}$, Xiangdong Liu² and Qiming Dai ${ }^{3^{*}}$

\begin{abstract}
Background: Hypertrophic cardiomyopathy (HCM) represents one of the most common inherited heart diseases. To identify key molecules involved in the development of HCM, gene expression patterns of the heart tissue samples in HCM patients from multiple microarray and RNA-seq platforms were investigated.

Methods: The significant genes were obtained through the intersection of two gene sets, corresponding to the identified differentially expressed genes (DEGs) within the microarray data and within the RNA-Seq data. Those genes were further ranked using minimum-Redundancy Maximum-Relevance feature selection algorithm. Moreover, the genes were assessed by three different machine learning methods for classification, including support vector machines, random forest and k-Nearest Neighbor.

Results: Outstanding results were achieved by taking exclusively the top eight genes of the ranking into consideration. Since the eight genes were identified as candidate HCM hallmark genes, the interactions between them and known HCM disease genes were explored through the protein-protein interaction (PPI) network. Most candidate $\mathrm{HCM}$ hallmark genes were found to have direct or indirect interactions with known HCM diseases genes in the PPI network, particularly the hub genes JAK2 and GADD45A.
\end{abstract}

Conclusions: This study highlights the transcriptomic data integration, in combination with machine learning methods, in providing insight into the key hallmark genes in the genetic etiology of HCM.

Keywords: Hypertrophic cardiomyopathy, Microarray, RNA-Seq, Classification, JAK2

\section{Background}

Hypertrophic cardiomyopathy (HCM) is a genetically heterogeneous cardiac muscle disorder characterized by left ventricle hypertrophy in the absence of abnormal loading conditions [1]. HCM occurs in at least 1 in 500 of the general population, making it one of the most common inherited heart diseases [2]. In $70 \%$ of HCM patients, the disease is caused by mutations in sarcomeric genes, Z-disc genes, calcium-handling genes and so

\footnotetext{
*Correspondence: qimingdai@icloud.com

${ }^{3}$ Department of Cardiology, ZhongDa Hospital, Southeast University, Nanjing, China

Full list of author information is available at the end of the article
}

on. The genetic background of about 30\% HCM patients remains unknown [3]. The cellular signaling processes that lead from the primary mutation to the HCM phenotype are also poorly understood. Therefore, it is essential to investigate the pathogenic mechanisms and develop novel diagnostic hallmark genes.

Two gene expression profiling technologies, microarray and RNA sequencing (RNA-Seq), have been widely used for obtaining gene expression signature. Compared to microarray, RNA-Seq can simultaneously detect whole gene expression levels [4]. Existing evidence showed a high consistency between microarray and RNA-Seq [5, 6]. 
In the last decade, various methods for classification have been developed and gained great attention of biomedical applications $[7,8]$. In most classification studies, support vector machines (SVM), random forest (RF), $\mathrm{K}$-Nearest-Neighbors (KNN) are reported as the foremost classifiers producing high accuracies [9].

In this study, the integrated analysis of transcriptomic datasets from different platforms was performed to identify differentially expressed genes (DEGs) between HCM patients and healthy controls (Fig. 1). Machine learning methods, including SVM, RF and KNN, were applied to prioritize the HCM candidate hallmark genes. This study provided novel perspective for understanding mechanism and exploiting new therapeutic means for HCM.

\section{Methods}

\section{Data collection}

Gene expression profiles of the heart tissue samples in HCM patients/mice and healthy controls and patientspecific induced pluripotent stem cells-derived cardiomyocytes (iPSC-CMs) were obtained from ArrayExpress (http://www.ebi.ac.uk/arrayexpress/), Gene Expression Omnibus (GEO, https://www.ncbi.nlm.nih.gov/gds/) and Sequence Read Archive (SRA, https://www.ncbi.nlm.nih. gov/sra/).

\section{Microarray data analysis}

For microarray datasets, standard analysis process including quality control, pre-processing, normalization using Limma and Lumi packages across Illumina and CapitalBio platforms was performed $[10,11]$. To avoid distortion of the results by noise, uninformative probes (low variance, expressed uniformly close to background detection levels) were filtered out. Finally, normalized $\log 2$-transformed expression values were obtained.

\section{RNA-seq data analysis}

For RNA-Seq data sets, after removing adapters and low-quality bases using the Trimmomatic program, we implemented STAR [12] to map reads to human genome hg38. Samtools [13] and Htseq [14] were then used to obtain the read count for each gene. Then the expression

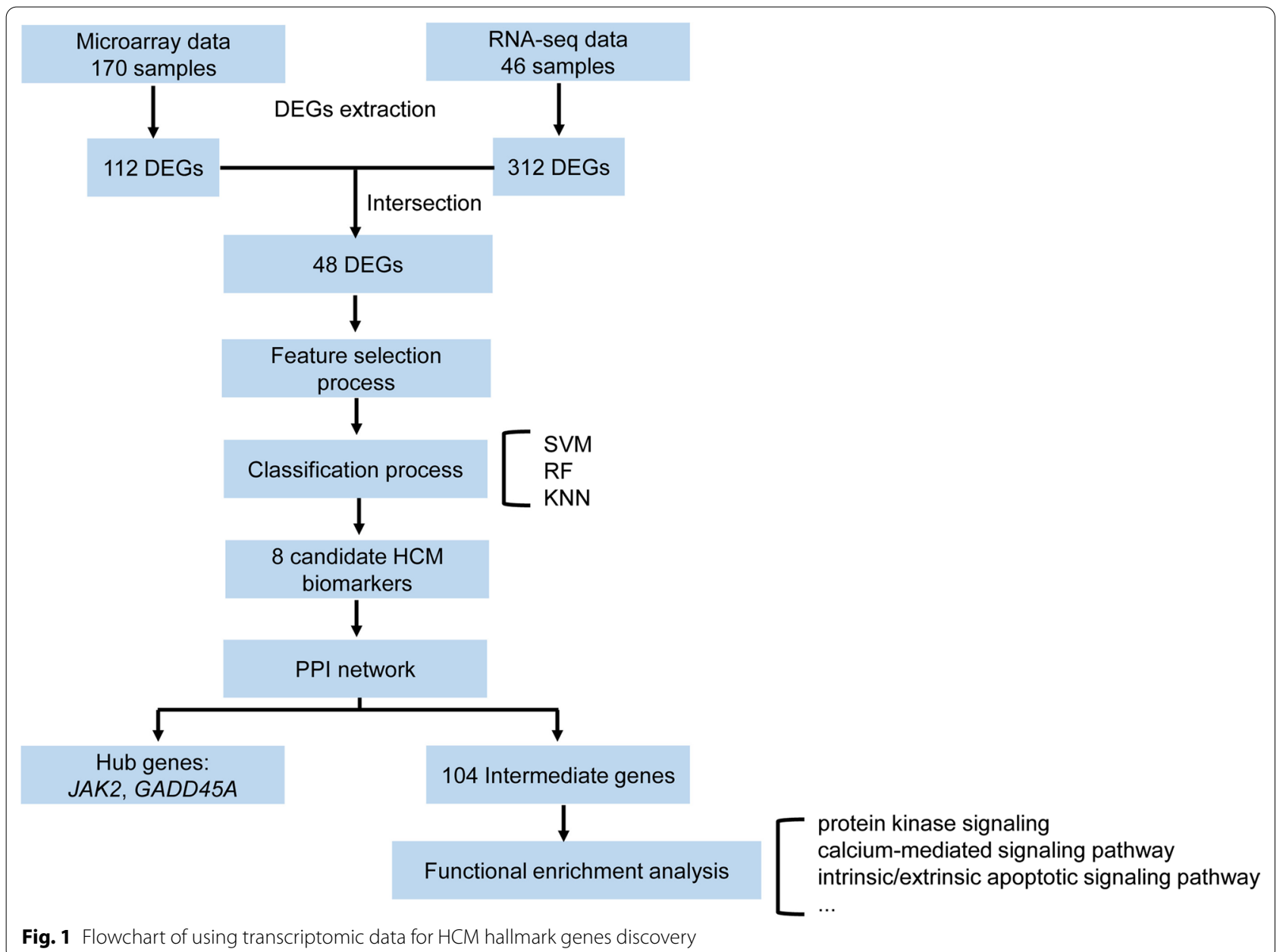


values for the genes were calculated using the Cqn and the NOISeq R packages $[15,16]$.

\section{DEGs extraction}

The expression values obtained from both microarray and RNA-Seq technologies were integrated using the merge function from the base $\mathrm{R}$ package. Extraction of DEGs was performed using the limma R package, at both individual level (microarray data and RNA-Seq data separately). Then a normalization of all joint data was applied using the NormalizedBetweenArrays function. Log-fold change (LFC) and adjusted p value (adj. PV) using Benjamini Hochberg's method, were considered to select statistically highly differentiated expressed genes.

\section{Feature selection process}

The feature selection process was performed to obtain a ranking of the most relevant DEGs, using the minimumRedundancy Maximum-Relevance (mRMR) algorithm [17]. To create this ranking, mRMR sorts the genes so that they bring largest relevance with respect to the class ( $\mathrm{HCM} /$ control), at the same time, they have lowest redundancy among themselves. Therefore, this algorithm will rank in first position the gene that contains the largest amount of information, but the following genes will provide also minimum redundancy (apart from maximum relevance as regard to the class) with respect to the already selected genes. The mRMR algorithm was implemented by importing the pymrmr package with python [18].

\section{Classification process}

In the classification process, three different machine learning algorithms, including SVM, RF and KNN, were implemented to assess the results. The experiments are implemented with Python using the svm, RandomForestClassifier, KNeighborsClassifier from scikit-learn libraries [19].

SVM are supervised learning models with associated learning algorithms that analyze data used for classification and regression analysis [20]. Four kernels, including the radial basis function (RBF), polynomial, linear and sigmoid kernel, were tested to implement the SVM algorithm. Among the four kernels, the RBF kernel showed a good performance and was chosen by using the argument (kernel $=$ 'rbf').

RF is essentially, an ensemble of decision trees combined where each tree votes on the class assigned to a given sample, with the most frequent answer winning the vote [21]. For the RF classifier, two main parameters were tested and evaluated: n_estimators and min_samples_leaf.
The KNN algorithm is an instance-based learning method for classifying objects based on closest training examples in the feature space [22]. Two main parameters $\mathrm{n} \_$neighbors and $\mathrm{p}$ were tested to find the optimal KNN model for classification.

Ten-fold cross-validation (CV) was used over the training dataset to obtain the optimal hyperparameters for the methodologies. Accuracy and f1-score were used as the performance measures.

\section{Protein interaction network}

The protein-protein interaction (PPI) network is represented as graphs where nodes and edges are proteins and pair wise interactions, respectively. Only intermediate genes known to interact between 47 known HCM disease genes and HCM candidate hallmark genes were included. Experimentally verified interaction data from StringDB [23] and Biogrid [24] were used for establishing the PPI network. Only medium- and high-confidence experimental interactions in StringDB were shown, although these may not always represent local interactions. Cytoscape (version 3.7.2), a bioinformatics software platform, was used for visualizing the molecular interaction networks [25]. ClueGO, a cytoscape plugin, was used for functional enrichment analysis based on the intermediate genes [26].

\section{Statistical analysis}

Statistical analysis and Pearson's correlation analysis were performed with R studio.

\section{Results}

Integration of samples

Two hundred sixteen samples in 5 datasets were selected, including $154 \mathrm{HCM}$ samples and 62 healthy control samples (Table 1). Four datasets contain gender information, and one of them with $M Y H 7 / M Y B P C 3$ genotype information. Both microarray and RNA-Seq data analysis were conducted and the gene expression values were obtained for each technology separately. The representation of the individual dataset reflected several different expression value ranges (Fig. 2). To remove dynamic expression variability between samples due to different platforms, a normalization of all joint data per technology was performed (Fig. 3).

\section{Detection of DEGs}

After data integration, the general LFC value of the identified DEGs were relatively low with a maximum value of 2.36. Therefore, the criteria for DEGs detection we chosen was less stringent, with $|\mathrm{LFC}| \geq 0.6$, and $\mid \mathrm{adj}$. $\mathrm{PV} \mid \leq 0.05$. Two sets of DEGs were identified for microarray dataset and RNA-Seq dataset (Fig. 1). A total of 48 
Table 1 Characteristics of microarray and RNA-Seq datasets in the study

\begin{tabular}{|c|c|c|c|c|c|c|}
\hline Datasets & Source & Type & Technology & Platform & Year & Case/Control \\
\hline E-GEOD-36961 & Human heart tissue & mRNA & Microarray & Illumina GPL15389 & 2012 & $106 / 39$ \\
\hline GSE32453 & Human heart tissue & mRNA & Microarray & Illumina GPL6104 & 2012 & $8 / 5$ \\
\hline E-GEOD-68316 & Human heart tissue & mRNA & Microarray & CapitalBio GPL20113 & 2015 & $7 / 5$ \\
\hline Integrated (Microarray) & & & & & & $121 / 49$ \\
\hline GSE89714 & Human heart tissue & mRNA & RNA-Seq & Illumina GPL20795 & 2016 & $5 / 4$ \\
\hline GSE130036 & Human heart tissue & mRNA & RNA-Seq & Illumina GPL11154 & 2019 & $28 / 9$ \\
\hline Integrated (RNA-Seq) & & & & & & $33 / 13$ \\
\hline
\end{tabular}

Source: GEO/ArrayExpress accession

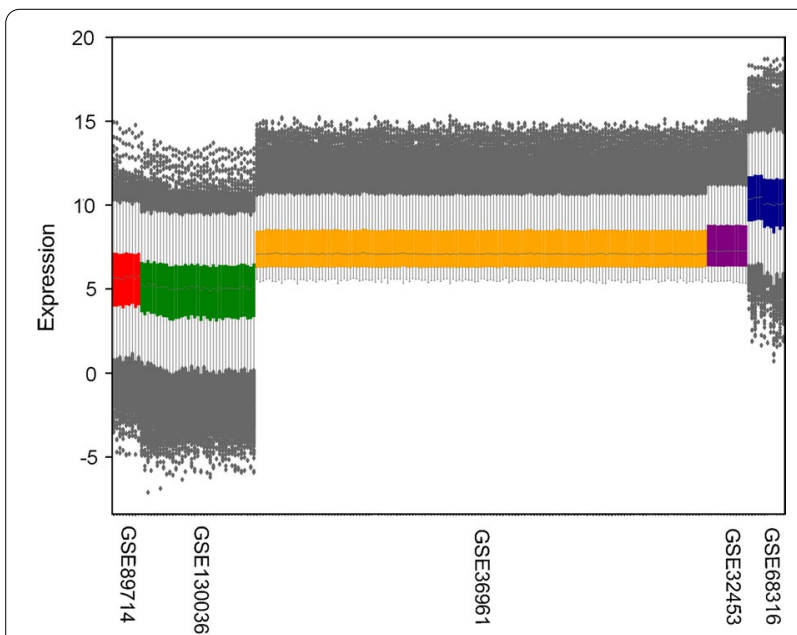

Fig. 2 Expression profile of each dataset before normalization

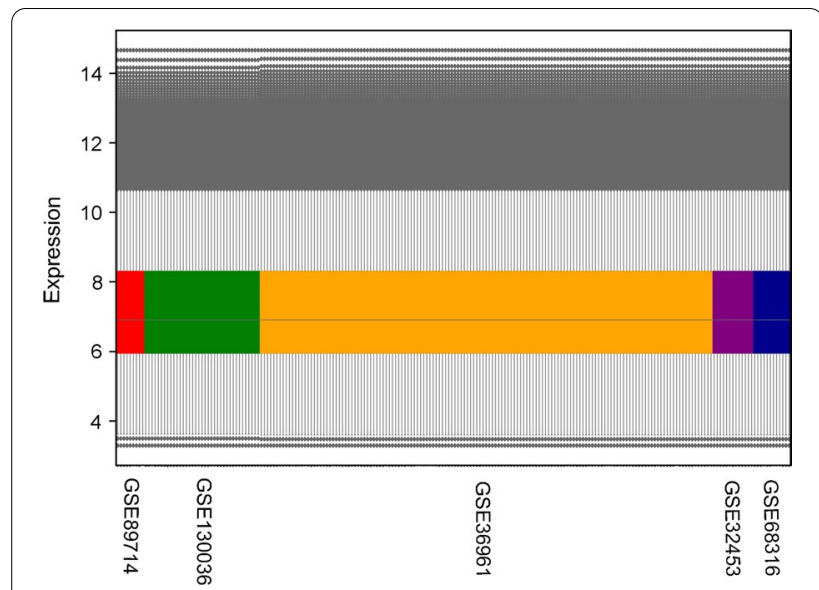

Fig. 3 Expression profile of each dataset after normalization

common DEGs were obtained through the intersection of the two sets of DEGs (Fig. 4). Two genes (GADD45B and THBS1) showed opposite direction in the two DEGs sets (Additional file 1: Table S1 and S2).

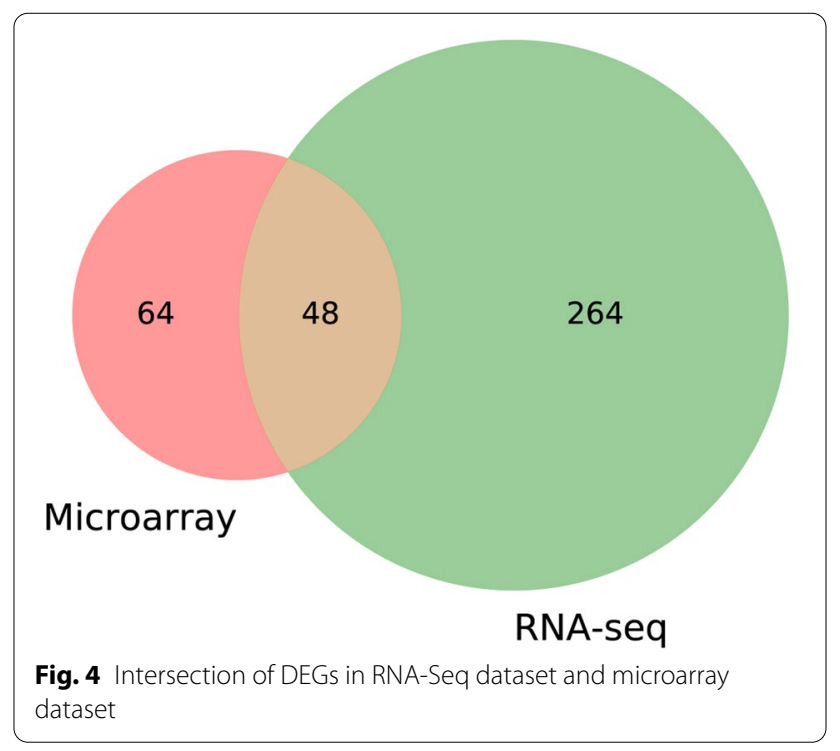

\section{Assessment of DEGs}

The feature selection process was applied to the 48 DEGs, and the ranking of the genes was based on its relevance with HCM using the mRMR algorithm. Subsequently, the performance of the obtained ranking was evaluated. Stratified sampling was used to divide the integrated dataset into a training dataset (172 samples) and a test dataset (44 samples). The expression values of the 48 DEGs were chosen as classification features. Three different classifiers were implemented and compared, including SVM [20], RF [21] and KNN [22]. Furthermore, the comparison has been performed for both accuracy and f1-score with different number of genes. The f1-score is a measure of a test's accuracy, calculated by using both the precision or accuracy and the recall or sensitivity.

The validation results (10-CV over the training dataset) and test results using the three classifiers were shown in Additional file 1: Table S3. These validation results were above $87 \%$ using only the first gene of the ranking for classification, and above $93 \%$ using a reduced set of eight genes in the ranking. Using those eight genes, the test 


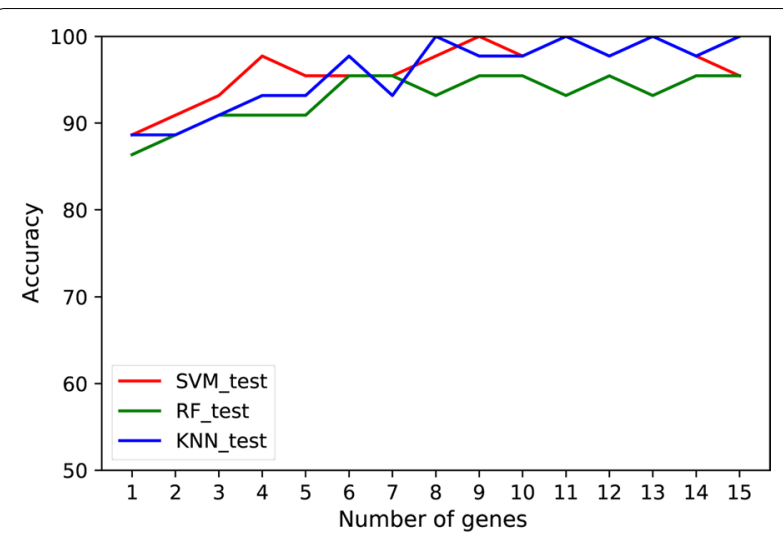

Fig. 5 The test accuracy achieved by SVM, RF and KNN using the most relevant genes obtained by mRMR. Similar trends can be observed for f1-score

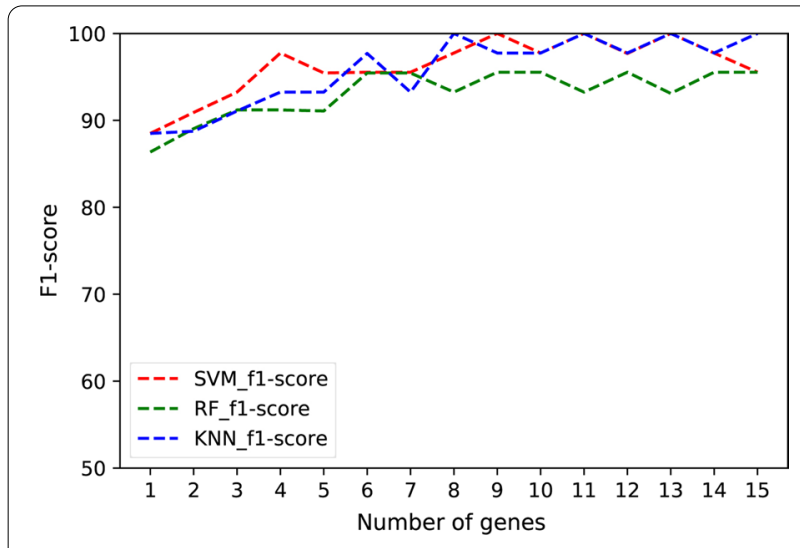

Fig. 6 The f1-score achieved by SVM, RF and KNN using the most relevant genes obtained by $\mathrm{mRMR}$ results showed an accuracy of $97.73 \%$ using SVMs, $100 \%$ using KNN, nevertheless lower using RF with a 93.18\%. Consequently, the main set of 48 DEGs was reduced to the eight genes, which allow discerning whether new samples are HCM or not. The eight genes $(J A K 2, C 1 R$, MS4A7, MBP, METTL7B, GADD45A, CD209, TRAK2) were then listed as candidate HCM hallmark genes.

Figures 5 and 6 showed the evolution of accuracy and f1-score for the three classifiers using a different number of genes. Regarding the three classifiers, SVM reached comparable results with KNN, better than RF. Expression levels of the eight candidate HCM hallmark genes were shown in Fig. 7, revealing a clear differentiation between the average value of the HCM and healthy control samples.

To see whether gender and $M Y H 7 / M Y B P C 3$ genotype affects the expression of the eight candidate HCM hallmark genes, comparisons of the expression values were performed using student's t-test. The results showed a significant difference in the expression of the eight genes between male HCM hearts and male control hearts, as well as between female HCM hearts and female control hearts, while no significant difference was noted between male and female HCM samples. Moreover, no significant difference in the expression of the eight genes was noted between MYH7/MYBPC3 genotype positive and negative HCM samples.

The expression of the $48 \mathrm{HCM}$ relevant genes was also explored in the iPSC-CMs from a family cohort carrying a hereditary HCM missense mutation (Arg663His) in the MYH7 gene (GSE35229). The expression of one candidate hallmark gene $M E T T L 7 B$ was significantly increased in iPSC-CMs compared with human embryonic stem cells (hESCs) and fibroblasts $(\mathrm{p}<0.01)$ [27].
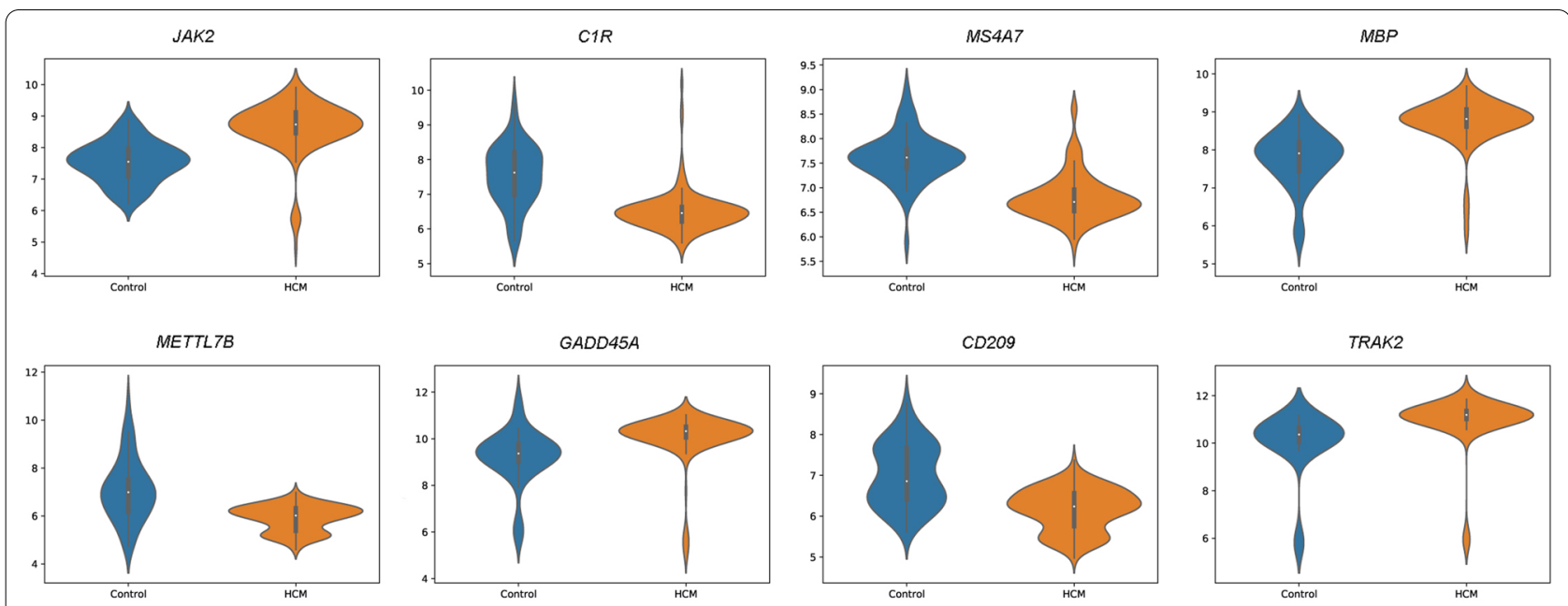

Fig. 7 Average expression value violin plots of the eight candidate HCM hallmark genes obtained in this study 


\section{Protein interaction network}

Since the eight genes were identified as candidate hallmark genes for HCM, the investigation of their interactions with known HCM disease genes would provide a deep insight into their biological roles. The interaction data were extracted from StringDB and Biogrid database $[23,24]$, and the PPI network was formed to summarize these links. As shown in Fig. 8, a total of 155 nodes and 463 edges relationship pairs were identified in the PPI network, including 44 known HCM disease genes, 7 candidate HCM hallmark genes and 104 intermediate genes. Four candidate HCM hallmark genes $(J A K 2, M B P$, $C D 209$, TRAK2) have both direct and indirect interactions with known HCM disease genes, while the other 3 candidate HCM hallmark genes (C1R, GADD45A, MET$T L 7 B)$ only have indirect interactions with known HCM disease genes. Among the eight candidate HCM hallmark genes, the most connected genes were $J A K 2$ and GADD45A, both with 35 underlying edges.

Further functional enrichment analysis showed that the intermediate genes were mostly transcription factors and protein kinases, which are involved in the regulation of multiple signaling transduction pathways, including protein kinase signaling pathway, calcium-mediated signaling pathway and intrinsic/extrinsic apoptotic signaling pathway, et al. Furthermore, intermediate genes that participate in positive regulation of cardiac muscle tissue growth and cardiac septum morphogenesis were also identified in the process.

\section{JAK2 and GADD45A}

The expression levels of $J A K 2$ and GADD45A with the well-established biomarkers of HCM (NPPA and $N P P B)$ were explored using the Pearson's correlation analysis. Positive relationship can be found between $J A K 2$ and NPPA $(\mathrm{r}=0.62, \mathrm{p}=4.05 \mathrm{e}-06), J A K 2$ and $N P P B(\mathrm{r}=0.65, \mathrm{p}=8.88 \mathrm{e}-07), G A D D 45 A$ and $N P P A$ $(\mathrm{r}=0.63, \mathrm{p}=2.18 \mathrm{e}-06), G A D D 45 A$ and NPPB $(\mathrm{r}=0.66$, $\mathrm{p}=5.70 \mathrm{e}-07)$.

The expression of $J A K 2$ were further explored in the heart tissues of HCM animal models. Significant difference was noted between $\mathrm{MHC}^{403 /+}$ mice and wild type (WT) mice based on the dataset GSE52038 $(\mathrm{p}<0.01)$ [28]. However, negative results were found in the other

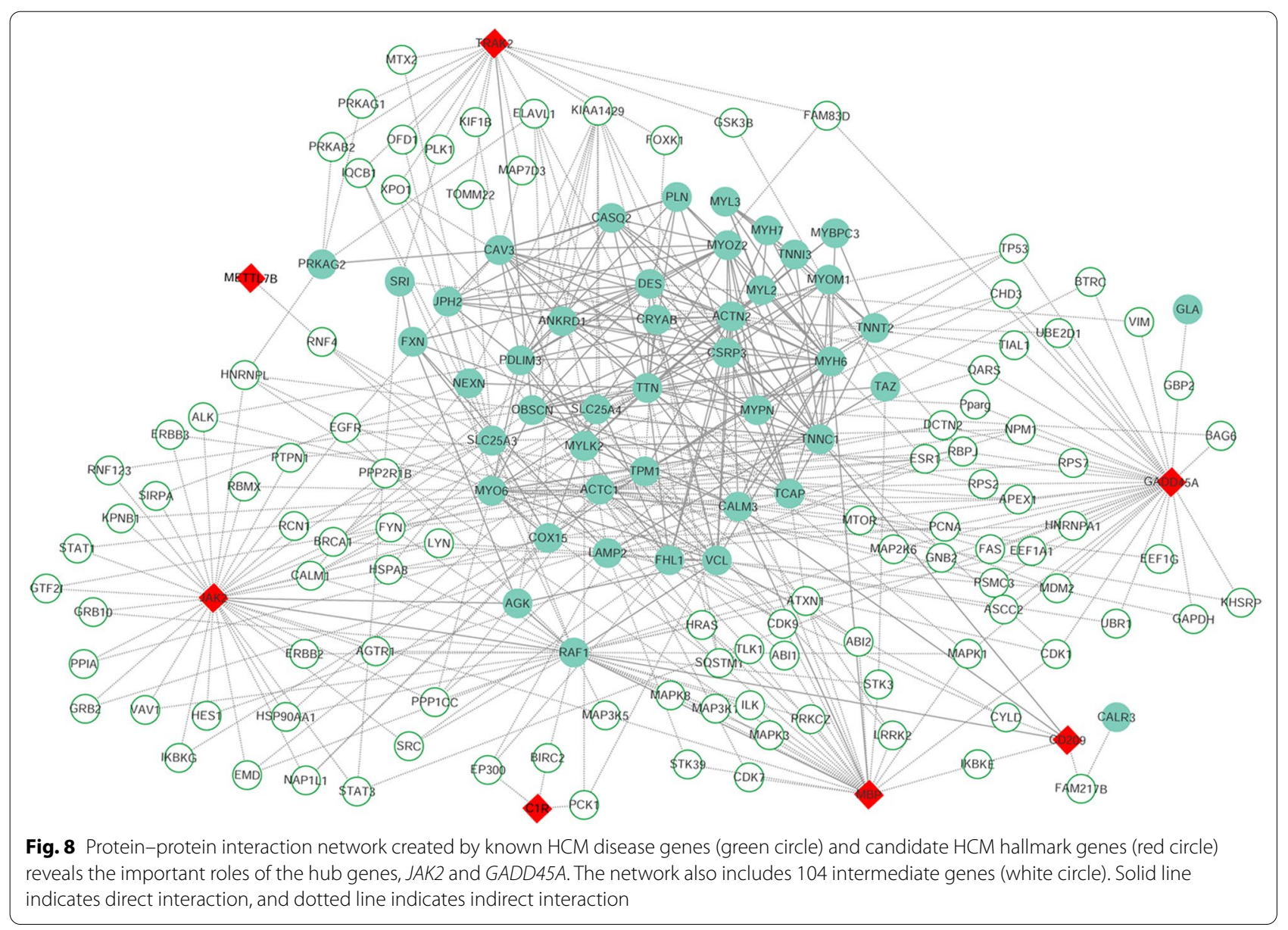


HCM animal models, probably due to the timing of the detection, since the samples from human were mostly collected in the end stage of HCM, whereas the samples from animal models were generally collected in the earlier disease stage of HCM.

\section{Discussion}

In the last decades, two gene expression profiling technologies including microarray and RNA-Seq have been proved to be excellent in revealing the biomarkers and cellular pathways of human disease [29]. Previous studies on HCM transcriptomic data have focused on only the microarray datasets or the RNA-Seq datasets [30,31]. Advances in bioinformatics and the increasing number of transcriptomic datasets have enabled a full exploration of the integrated transcriptomic data to reveal molecular mechanisms underlying $\mathrm{HCM}$.

An exhaustive search from the GEO, ArrayExpress, and SRA public repository has been performed to collect HCM and control heart tissue samples from both technologies. After data integration and DEGs extraction, the general LFC value of the identified DEGs were relatively low, we assume that the mild change of gene expression may be related to the slow disease progression of most $\mathrm{HCM}$ cases.

During the classification process, SVM, RF and KNN technologies were implemented for the DEGs evaluation. The differences in performance among classification techniques are usual in this type of problems, and several papers comparing classification techniques for biological data can be found in the literature [32-34]. In the results above-mentioned, SVM classifier attains an optimal performance using only 8 genes. The behavior is also seen in the KNN technique, although with a lower performance. RF classifier obtained similar results when using the complete set of 48 genes but fails to design a simpler classifier with a low number of genes with optimal performance [32]. Thus, these results support the design of an optimal classifier based on SVM classifier with only eight genes.

The PPI network established between known HCM disease genes and eight HCM candidate hallmark genes contains helpful information for understanding the role of them in the development of HCM. JAK2 and GADD45A were found to be hub genes in the PPI network, indicating their important roles underlying HCM. Further functional enrichment analysis also showed that some intermediate genes participate in positive regulation of cardiac muscle tissue growth and cardiac septum morphogenesis.

Janus kinase 2, encoded by $J A K 2$, is a protein tyrosine kinase involved in a specific subset of cytokine receptor signaling pathways. As a member of JAK family, JAK2 is an important component in the Janus kinase/signal transducer and activator of transcription (JAK/STAT) signaling pathway. The JAK/STAT signaling triggers multiple signals involved in development, homeostasis and inflammation [35, 36]. Accumulating evidence indicated that the JAK/STAT signaling pathway played a vital role in transducing stress and growth signals in the hypertrophic heart $[37,38]$. The JAK/STAT pathway also transduces signals for a wide array of cytokines and growth factors including ANGII, TNF- $\alpha$, IL- $1 \beta$, IL- 6 and IFN- $\gamma$, all of which have been involved in cardiac hypertrophy [39-42]. Moreover, JAK2 has previously been reported to play an important role in left ventricular remodeling during pressure overload hypertrophy, and the development of hypertrophy can be blocked by pharmacological inhibition of JAK2 kinase [43]. Furthermore, one mutation V617F in JAK2 has been identified in one patient with myeloproliferative disorder (MPD) and HCM, suggesting a potential causative role of JAK2 in the development of HCM phenotype [44]. Recent studies also showed that cardiac JAK2 was critical for maintaining normal heart function, and its ablation produced a severe pathologic phenotype composed of myocardial remodeling [45]. Taken together, it is likely that $J A K 2$ plays a central role in the pathogenesis of HCM. From our previous study, rare mutations in JAK2 were identified in 9/72 (12.5\%) HCM patients without mutations in known HCM disease genes (Table 2) [3]. It would be interesting to further explore the specific role of these mutations and their associations with HCM.

Another hub gene GADD45A, encoding growth arrest and DNA damage inducible alpha, is a member of GADD45 gene family, which have been implicated in stress signaling responses to various physiological or environmental stressors, thus contributing to the maintenance of genomic stability [46]. Several previous studies have evaluated the hypothesis that two other GADD45 isoforms, including GADD45G and GADD45B, may have relevance to cardiac physiopathology $[47,48]$.

As one candidate hallmark gene, METTL7B is a member of mammalian methyltransferase-like family. The expression of METTL7B in HCM significantly decreased in our study. In line with our findings, one recent study showed that the expression levels of METTL7B in the

Table 2 Mutations in JAK2 (NM_008413) identified in 72 nonsarcomeric HCM patients

\begin{tabular}{lllll}
\hline Sample ID & Het/hom & AA change & SNP & MAF \\
\hline H13, H53, H44, H07 & het & N1108S & rs142269166 & $1.96 \mathrm{e}-3$ \\
$\mathrm{H} 17$ & het & L892V & rs201551707 & $6.56 \mathrm{e}-4$ \\
$\mathrm{H} 24, \mathrm{H} 51$ & het & L393V & rs2230723 & $7.88 \mathrm{e}-3$ \\
$\mathrm{H} 60, \mathrm{H} 65$ & het & R1063H & rs41316003 & $4.37 \mathrm{e}-3$ \\
\hline
\end{tabular}

$M A F$ minor allele frequency in GnomAD database 
cardiac tissue in the diabetic cardiomyopathy patient group were statistically lower than those in the healthy group [49]. However, the expression of METTL7B was significantly increased in iPSC-CMs compared with hESCs and fibroblasts. Despite the opposite results, all the present data support the importance of METTL7B in $\mathrm{HCM}$, experimental data have yet to be fully investigated to determine its pathogenic relevance.

Additionally, the list of HCM-related genes between our study and previous studies of those datasets were compared and found that even though some genes appeared in the opposite direction in separated datasets $[30,50]$, most HCM relevant genes showed the same directions between microarray and RNA-seq datasets, including the eight candidate HCM hallmark genes.

Furthermore, due to the limited number of genes detected in the microarray datasets compared to the RNA-seq datasets, focusing on common DEGs through the intersection of datasets tend to lose some important information that RNA-seq would confer. Unfortunately, no enriched biological function and pathway based on the 48 identified HCM relevant genes can be found through GO and pathway analysis. However, we are confident with the results because they have been validated in different platforms and different patient cohorts.

Previous studies have demonstrated that distinct cellular pathways were involved in the development of HCM corresponding to different causative gene mutations [40]. However, based on the results in this study, we assumed that the eight candidate hallmark genes may act as a central role in the mutual cellular pathways underlying the HCM phenotype, which can somehow be triggered by most causative gene mutation. Further studies are needed to decipher the specific role of the candidate hallmark genes associated with HCM.

\section{Conclusions}

Integrating transcriptomic datasets from different platforms, have greatly aid the utility of biological data and improved the interpretation of gene expression values. Our results showed that the pipeline has good performance and a high accuracy of the classifier to distinguish unknown samples. Additionally, the central role of JAK2 and GADD45A in the pathogenic mechanism of HCM was highlighted. These findings will greatly contribute to extending our knowledge of the biological changes underlying $\mathrm{HCM}$ and providing perspective to reveal the pathology and develop therapeutic targets for HCM.

\section{Supplementary Information}

The online version contains supplementary material available at https://doi. org/10.1186/s12872-021-02147-7.

Additional file 1. Table S1: The statistical information of the 48 DEGs obtained in microarray dataset. Table S2: The statistical information of the 48 DEGs obtained in RNA-Seq dataset. Table S3: Results of the three classifiers for both accuracy and f1-score when using different numbers of genes.

\section{Acknowledgements}

We would like to express our gratitude to the patients who agreed to participate in this study.

\section{Authors' contributions \\ JX and QM. D designed the study and wrote the manuscript. JX performed the computations and verified the analytical methods. QM. D and XD. L helped supervise the project. All authors discussed the results and contributed to the final manuscript.}

\section{Funding}

This work was supported by grant from Natural Science Foundation of Jiangsu Province (BK20180376).

\section{Availability of data and materials}

The datasets used and analyzed during the current study are available from three public database, ArrayExpress (http://www.ebi.ac.uk/arrayexpress/), Gene Expression Omnibus (GEO, https://www.ncbi.nlm.nih.gov/gds/) and Sequence Read Archive (SRA, https://www.ncbi.nlm.nih.gov/sra/). Accession numbers of the datasets used are shown in Table 1. The code for processing the data in the study is available within fgshare (https://doi.org/10.6084/m9. figshare.14650536).

\section{Declarations}

\section{Ethics approval and consent to participate}

The research with human subjects was approved by the ethics committee of Southeast University, China and was carried out in accordance with the guidelines of the Declaration of Helsinki. Informed consent was obtained from all the participants. The research with mice models gained approval from the ethics committee of Southeast University, China. Mice were sampled and managed in accordance with the National Institute of Health.

Consent for publication

Not applicable.

Competing interests

The authors declare that they have no competing interests.

\section{Author details}

${ }^{1}$ Department of Clinical Laboratory, ZhongDa Hospital, Southeast University, Nanjing, China. ${ }^{2}$ Institute of Life Science, Southeast University, Nanjing, China. ${ }^{3}$ Department of Cardiology, ZhongDa Hospital, Southeast University, Nanjing, China.

Received: 19 October 2020 Accepted: 17 June 2021

Published online: 06 July 2021 


\section{References}

1. Elliott PM, Anastasakis A, Borger MA, Borggrefe M, Cecchi F, Charron P, Hagege AA, Lafont A, Limongelli G, Mahrholdt H, et al. 2014 ESC Guidelines on diagnosis and management of hypertrophic cardiomyopathy: the Task Force for the Diagnosis and Management of Hypertrophic Cardiomyopathy of the European Society of Cardiology (ESC). EUR HEART J. 2014:35(39):2733-79.

2. Ingles J, Burns C, Bagnall RD, Lam L, Yeates L, Sarina T, Puranik R, Briffa T, Atherton JJ, Driscoll T, et al. Nonfamilial hypertrophic cardiomyopathy: Prevalence, natural history, and clinical implications. Circ Cardiovasc Genet. 2017;10:2

3. Xu J, Li Z, Ren X, Dong M, Li J, Shi X, Zhang Y, Xie W, Sun Z, Liu X, et al. Investigation of pathogenic genes in Chinese sporadic hypertrophic cardiomyopathy patients by whole exome sequencing. Sci Rep. 2015;5:16609.

4. Casamassimi A, Federico A, Rienzo M, Esposito S, Ciccodicola A. Transcriptome profiling in human diseases: new advances and perspectives. Int $J$ Mol Sci. 2017;18:8.

5. Castillo D, Galvez JM, Herrera LJ, Roman BS, Rojas F, Rojas I. Integration of RNA-Seq data with heterogeneous microarray data for breast cancer profiling. BMC Bioinformatics. 2017;18(1):506.

6. Nookaew I, Papini M, Pornputtapong N, Scalcinati G, Fagerberg L, Uhlen $\mathrm{M}$, Nielsen J. A comprehensive comparison of RNA-Seq-based transcriptome analysis from reads to differential gene expression and crosscomparison with microarrays: a case study in Saccharomyces cerevisiae. Nucleic Acids Res. 2012;40(20):10084-97.

7. Murugan A, Nair S, Kumar K. Detection of skin cancer using SVM, random forest and kNN classifiers. J Med Syst. 2019;43(8):269.

8. Wei L, Su R, Wang B, Li X, Zou Q, Gao X. Integration of deep feature representations and handcrafted features to improve the prediction of N6-methyladenosine sites. Neurocomputing. 2019:324:3-9.

9. Boateng EY, Otoo J, Abaye D. Basic tenets of classification algorithms K-nearest-neighbor, support vector machine, random forest and neural network: a review. J Data Anal Inf Process. 2020;08:341-57.

10. Ritchie ME, Phipson B, Wu D, Hu Y, Law CW, Shi W, Smyth GK. limma powers differential expression analyses for RNA-sequencing and microarray studies. Nucleic Acids Res. 2015;43(7):e47.

11. Du P, Kibbe WA, Lin SM. lumi: a pipeline for processing Illumina microarray. Bioinformatics. 2008;24(13):1547-8.

12. Dobin A, Davis CA, Schlesinger F, Drenkow J, Zaleski C, Jha S, Batut $P$, Chaisson M, Gingeras TR. STAR: ultrafast universal RNA-seq aligner. Bioinformatics. 2013:29(1):15-21.

13. Li H, Handsaker B, Wysoker A, Fennell T, Ruan J, Homer N, Marth G, Abecasis G, Durbin R. The Sequence Alignment/Map format and SAMtools. Bioinformatics. 2009;25(16):2078-9.

14. Anders S, Pyl PT, Huber W. HTSeq-a Python framework to work with highthroughput sequencing data. Bioinformatics. 2015:31(2):166-9.

15. Tarazona S, Furio-Tari P, Turra D, Pietro AD, Nueda MJ, Ferrer A, Conesa A. Data quality aware analysis of differential expression in RNA-seq with NOISeq R/Bioc package. Nucleic Acids Res. 2015;43(21):e140.

16. Hansen $\mathrm{KD}$, Irizarry RA, Wu Z. Removing technical variability in RNA-seq data using conditional quantile normalization. Biostatistics. 2012:13(2):204-16.

17. Ding $\mathrm{C}$, Peng $\mathrm{H}$. Minimum redundancy feature selection from microarray gene expression data. J Bioinform Comput Biol. 2005;3(2):185-205.

18. Peng $H$, Long F, Ding $C$. Feature selection based on mutual information: criteria of max-dependency, max-relevance, and min-redundancy. IEEE Trans Pattern Anal Mach Intell. 2005:27(8):1226-38.

19. Pedregosa F, Varoquaux G, Gramfort A, Michel V, Thirion B, Grisel O, Blondel M, Prettenhofer P, Weiss R, Dubourg V, et al. Scikit-learn: machine learning in python. J Mach Learn Res. 2011;12((null)):2825-30

20. Noble WS. What is a support vector machine? Nat Biotechnol. 2006;24(12):1565-7.

21. Diaz-Uriarte R, Alvarez DAS. Gene selection and classification of microarray data using random forest. BMC Bioinformatics. 2006;7:3.

22. Parry RM, Jones W, Stokes TH, Phan JH, Moffitt RA, Fang H, Shi L, Oberthuer A, Fischer M, Tong W, et al. k-Nearest neighbor models for microarray gene expression analysis and clinical outcome prediction. Pharmacogenomics J. 2010;10(4):292-309.

23. Szklarczyk D, Morris JH, Cook H, Kuhn M, Wyder S, Simonovic M, Santos A, Doncheva NT, Roth A, Bork P, et al. The STRING database in 2017: quality-controlled protein-protein association networks, made broadly accessible. Nucleic Acids Res. 2017;45(D1):D362-8.

24. Oughtred R, Stark C, Breitkreutz BJ, Rust J, Boucher L, Chang C, Kolas N, O'Donnell L, Leung G, McAdam R, et al. The BioGRID interaction database: 2019 update. Nucleic Acids Res. 2019;47(D1):D529-41.

25. Shannon P, Markiel A, Ozier O, Baliga NS, Wang JT, Ramage D, Amin $\mathrm{N}$, Schwikowski B, Ideker T. Cytoscape: a software environment for integrated models of biomolecular interaction networks. Genome Res. 2003;13(11):2498-504.

26. Bindea G, Mlecnik B, Hackl H, Charoentong P, Tosolini M, Kirilovsky A, Fridman WH, Pages F, Trajanoski Z, Galon J. ClueGO: a cytoscape plug-in to decipher functionally grouped gene ontology and pathway annotation networks. Bioinformatics. 2009;25(8):1091-3.

27. Lan F, Lee AS, Liang P, Sanchez-Freire V, Nguyen PK, Wang L, Han L, Yen $M$, Wang $Y$, Sun $N$, et al. Abnormal calcium handling properties underlie familial hypertrophic cardiomyopathy pathology in patient-specific induced pluripotent stem cells. Cell Stem Cell. 2013;12(1):101-13.

28. Christodoulou DC, Wakimoto H, Onoue K, Eminaga S, Gorham JM, DePalma SR, Herman DS, Teekakirikul P, Conner DA, McKean DM, et al. 5'RNA-Seq identifies Fhl1 as a genetic modifier in cardiomyopathy. J Clin Invest. 2014;124(3):1364-70.

29. Ibrahim NE, Januzzi JL Jr. Established and emerging roles of biomarkers in heart failure. Circ Res. 2018;123(5):614-29.

30. Liu X, Ma Y, Yin K, Li W, Chen W, Zhang Y, Zhu C, Li T, Han B, Liu X, et al. Long non-coding and coding RNA profiling using strand-specific RNAseq in human hypertrophic cardiomyopathy. Sci Data. 2019;6(1):90.

31. Li J, Wu Z, Zheng D, Sun Y, Wang S, Yan Y. Bioinformatics analysis of the regulatory IncRNAmiRNAmRNA network and drug prediction in patients with hypertrophic cardiomyopathy. Mol Med Rep. 2019;20(1):549-58.

32. Statnikov A, Wang $L$, Aliferis CF. A comprehensive comparison of random forests and support vector machines for microarray-based cancer classification. BMC Bioinformatics. 2008;9:319.

33. Statnikov A, Aliferis CF. Are random forests better than support vector machines for microarray-based cancer classification? AMIA Annu Symp Proc. 2007:2007:686-90.

34. Wu W, Xing EP, Myers C, Mian IS, Bissell MJ. Evaluation of normalization methods for cDNA microarray data by k-NN classification. BMC Bioinformatics. 2005;6:191.

35. Moresi V, Adamo S, Berghella L. The JAK/STAT pathway in skeletal muscle pathophysiology. Front Physiol. 2019;10:500.

36. O'Shea JJ, Schwartz DM, Villarino AV, Gadina M, McInnes IB, Laurence A. The JAK-STAT pathway: impact on human disease and therapeutic intervention. Annu Rev Med. 2015;66:311-28.

37. Fan Z, Gao Y, Huang Z, Xue F, Wu S, Yang J, Zhu L, Fu L. Protective effect of hydrogen-rich saline on pressure overload-induced cardiac hypertrophyin rats: possible role of JAK-STAT signaling. BMC Cardiovasc Disord. 2018;18(1):32.

38. Wagner MA, Siddiqui MA. The JAK-STAT pathway in hypertrophic stress signaling and genomic stress response. JAKSTAT. 2012;1(2):131-41.

39. Eid RA, Alkhateeb MA, El-Kott AF, Eleawa SM, Zaki M, Alaboodi SA, Salem AA, Aldera $\mathrm{H}$, Alnamar NM, Alassiri M, et al. A high-fat diet rich in corn oil induces cardiac fibrosis in rats by activating JAK2/STAT3 and subsequent activation of ANG II/TGF-1 beta/Smad3 pathway: the role of ROS and IL-6 trans-signaling. J Food Biochem. 2019;43(8):12952.

40. Nakamura M, Sadoshima J. Mechanisms of physiological and pathological cardiac hypertrophy. Nat Rev Cardiol. 2018;15(7):387-407.

41. Terrell AM, Crisostomo PR, Wairiuko GM, Wang M, Morrell ED, Meldrum DR. Jak/STAT/SOCS signaling circuits and associated cytokine-mediated inflammation and hypertrophy in the heart. Shock. 2006;26(3):226-34.

42. Schieffer B, Luchtefeld M, Braun S, Hilfiker A, Hilfiker-Kleiner D, Drexler $H$. Role of NAD(P)H oxidase in angiotensin II-induced JAK/STAT signaling and cytokine induction. Circ Res. 2000;87(12):1195-201.

43. Beckles DL, Mascareno E, Siddiqui MA. Inhibition of Jak2 phosphorylation attenuates pressure overload cardiac hypertrophy. Vascul Pharmacol. 2006;45(6):350-7

44. Gattenlohner S, Ertl G, Einsele H, Kircher S, Muller-Hermelink HK, Marx A. Cardiac JAK2 mutation V617F in a patient with cardiomyopathy and myeloproliferative disease. Ann Intern Med. 2008;149(1):69-71.

45. Gan XT, Rajapurohitam V, Xue J, Huang C, Bairwa S, Tang X, Chow JT, Liu MF, Chiu F, Sakamoto K, et al. Myocardial hypertrophic remodeling and 
impaired left ventricular function in mice with a cardiac-specific deletion of Janus Kinase 2. Am J Pathol. 2015;185(12):3202-10.

46. Liebermann DA, Hoffman B. Gadd45 in stress signaling. J Mol Signal. 2008:3:15.

47. Lucas A, Mialet-Perez J, Daviaud D, Parini A, Marber MS, Sicard P. Gadd45gamma regulates cardiomyocyte death and post-myocardial infarction left ventricular remodelling. Cardiovasc Res. 2015;108(2):254-67.

48. Wang J, Wang $H$, Chen J, Wang $X$, Sun $K$, Wang $Y$, Wang J, Yang $X$, Song $X$, $X$ in $Y$, et al. GADD45B inhibits MKK7-induced cardiac hypertrophy and the polymorphisms of GADD45B is associated with inter-ventricular septum hypertrophy. Biochem Biophys Res Commun. 2008;372(4):623-8.
49. Li N, Wu H, Geng R, Tang Q. Identification of Core Gene Biomarkers in Patients with Diabetic Cardiomyopathy. Dis Markers. 2018;2018:6025061.

50. Yang W, Li Y, He F, Wu H. Microarray profiling of long non-coding RNA (IncRNA) associated with hypertrophic cardiomyopathy. BMC Cardiovasc Disord. 2015;15:62.

\section{Publisher's Note}

Springer Nature remains neutral with regard to jurisdictional claims in published maps and institutional affiliations.
Ready to submit your research? Choose BMC and benefit from:

- fast, convenient online submission

- thorough peer review by experienced researchers in your field

- rapid publication on acceptance

- support for research data, including large and complex data types

- gold Open Access which fosters wider collaboration and increased citations

- maximum visibility for your research: over $100 \mathrm{M}$ website views per year

At BMC, research is always in progress.

Learn more biomedcentral.com/submissions 